\title{
Spectroscopy of Explosive Materials in the THz Range
}

\author{
N. PALKA* \\ Institute of Optoelectronics, Military University of Technology, S. Kaliskiego 2, 00-908 Warsaw, Poland \\ Absorption spectra of hexogen and penthrite- 2 commonly used explosive materials in the range $0.3-3 \mathrm{THz}$ are \\ presented. The measurements were carried out by means of time domain spectroscopy and compared to spectra \\ obtained by Fourier transform infrared spectroscopy. Good conformity is observed.
}

PACS numbers: 07.57.Ty, 07.57.Pt, 78.20.Ci, 78.40.Pg

\section{Introduction}

Development and implementation of terahertz (0.1-10 THz) technology in security area is connected with unique features of terahertz radiation. Frequency of terahertz radiation corresponds well with frequency of a normal mode (vibrational and rotational) of oscillating molecules of chemical compounds in room temperature and acoustic vibrations of molecules. Accordingly, chemical compounds of dangerous materials have specific absorption and emission spectra in terahertz range, which allows us to identify them. Therefore, many explosives (e.g., hexogen, penthrite, trinitrotoluene, octogen) and drugs have characteristic transmission/ reflection features in the $\mathrm{THz}$ range that could be distinguishable from other common materials like clothes, human skin or metal materials.

Moreover, terahertz waves penetrate many materials apart from metals or polar mediums (like water). Therefore, terahertz radiation can scan through packing, clothes, shoes, bags and is suitable for screening baggage and primarily for looking for materials hidden on human body under coverings. Such advantages are of great importance for security and defense related applications.

Spectra of pure explosives in $\mathrm{THz}$ range have been measured by many groups [1-5]. However, spectra of the same material can differ because of different sample preparation process. One should takes into account sizes of particles and a material which is used to dissolve explosives (usually teflon or HDPE). Moreover, some differences in spectra are observed because of different measurement techniques (time domain spectroscopy (TDS) versus Fourier transform infrared (FTIR) spectroscopy) and water vapor influences.

In this paper we report on measurements absorption spectra of hexogen (RDX) and penthrite (PETN) in the range $0.3-3 \mathrm{THz}$. The measurements were carried out by means of TDS and compared to spectra obtained by FTIR. Only small discrepancies are observed. The obtained characteristics can be used to create a data base of spectra of explosive materials.

* e-mail: npalka@wat.edu.pl

\section{Sample preparation}

Sample preparation is a very important factor to obtain reliable spectra. If the particle size of solid material is comparable to the $\mathrm{THz}$ wavelength $(100-500 \mu \mathrm{m})$, the scattering may cause a significant change in spectrum of measured sample [1]. Moreover, attenuation of pure explosives is too high to obtain reliable measurements in $\mathrm{THz}$ range, so $10 \%$ of explosives were mixed with $90 \%$ of teflon (PTFE) to obtain $1 \mathrm{~g}$ of mixture. Next, it was mixed with ethanol to get even mixture and dried through 30 min. Afterwards, the powder was weighted to obtain $400 \mathrm{mg}$ samples, loaded to a form and pressed with a pressure of about $100 \mathrm{MPa}$. As a result, a pellet with diameter of $13 \mathrm{~mm}$ and thickness of about $1.4 \mathrm{~mm}$ was obtained. As a reference, a pellet made of pure teflon with the same dimensions was prepared.

\section{Setup}

Time domain spectrometry setup (from EKSPLA) with pulses generated by an $800 \mathrm{~nm}$ femtosecond laser (Fusion20, Femtolaser, a pulse duration of 20 fs, repetition rate of $80 \mathrm{MHz}$, and average power of $200 \mathrm{~mW}$ ) was used - Fig. 1. The laser beam was split into a pump and probe and directed through a system of mirrors to an emitter and a detector. Low temperature grown GaAs dipole antennas with gap distance of about $6 \mu \mathrm{m}$ were applied as the emitter and the detector. The pump beam was focused on the biased emitter antenna to generate $\mathrm{THz}$ pulses through a photoconductive phenomenon. The emitted $\mathrm{THz}$ pulses were collimated by a

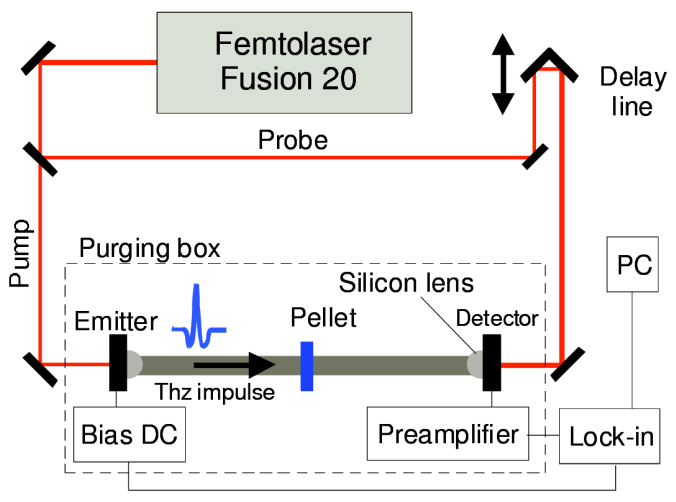

Fig. 1. Time domain spectrometry setup. 
silicon lens. The pellets were placed in the middle of the distance between the emitter and detector, perpendicularly to the incident $\mathrm{THz}$ beam. The transmitted $\mathrm{THz}$ beam was detected by means of the detector antenna gated using the laser probe beam and a mechanical delay line. A lock-in amplifier and LabView-based software was used to collect and process data. The system was purged with dry nitrogen gas to eliminate water vapor.

\section{Results and discussion}

Figure 2a (3a) presents two THz impulses - measured with the pure teflon sample [1-6] and with the pellet which consists of mixture of teflon and RDX (PETN). Figure $2 \mathrm{~b}(3 \mathrm{~b})$ shows the $\mathrm{THz}$ spectra, ranging between 0.3 and $3 \mathrm{THz}$, obtained by applying a fast Fourier transform (FFT) to the $\mathrm{THz}$ impulses.
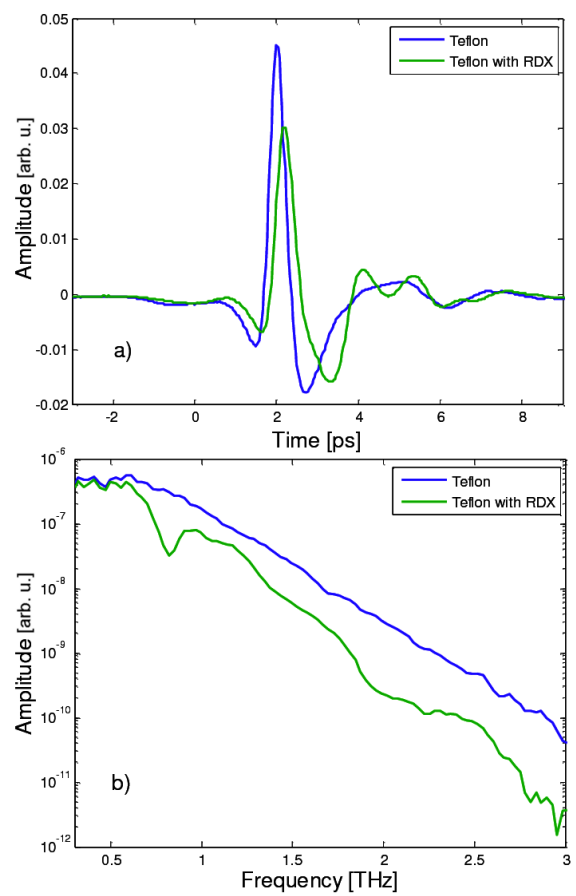

Fig. 2. THz impulses measured for teflon and teflon-RDX pellets (a), FFT of the impulses (b).

It is clearly seen in Figs. 2 and 3 that explosives in the pellets change the shape of the impulses as well as spectra (FFT modules). A time shift between impulses in Fig. 2a and $2 \mathrm{~b}$ is connected with the fact that samples with explosives have slightly bigger refractive indices, which increases optical path inside the pellets. This shift can also result from the fact that the pellets can differ in thickness because of inaccurate preparation process.

Next, attenuation can be determined as a ratio between spectra of teflon and teflon-RDX (PETN) samples - Fig. 4. Attenuation spectra of the same pellets measured by means of FTIR (Bruker 113) are also presented for verification.
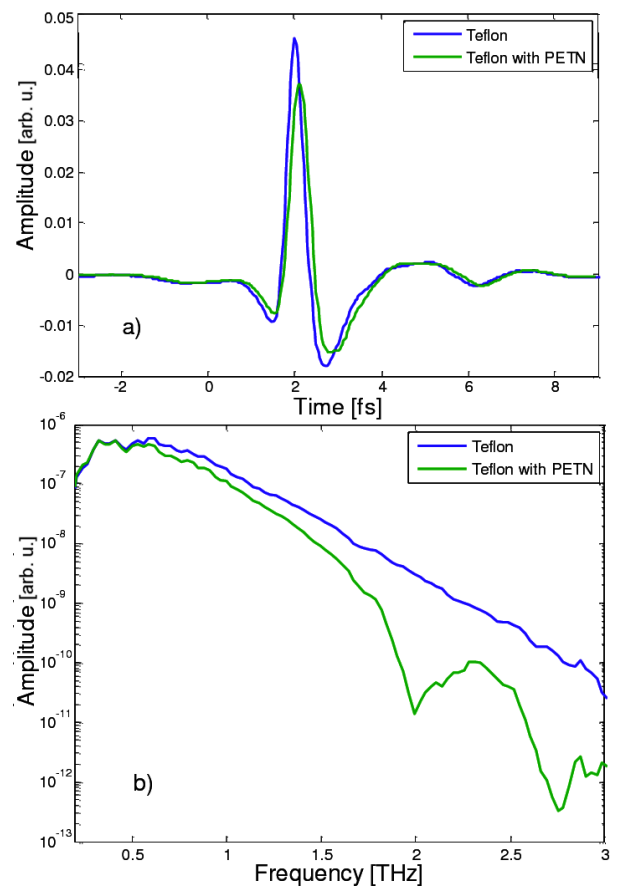

Fig. 3. THz impulses measured for teflon and teflon-PETN pellets (a), FFT of the impulses (b).
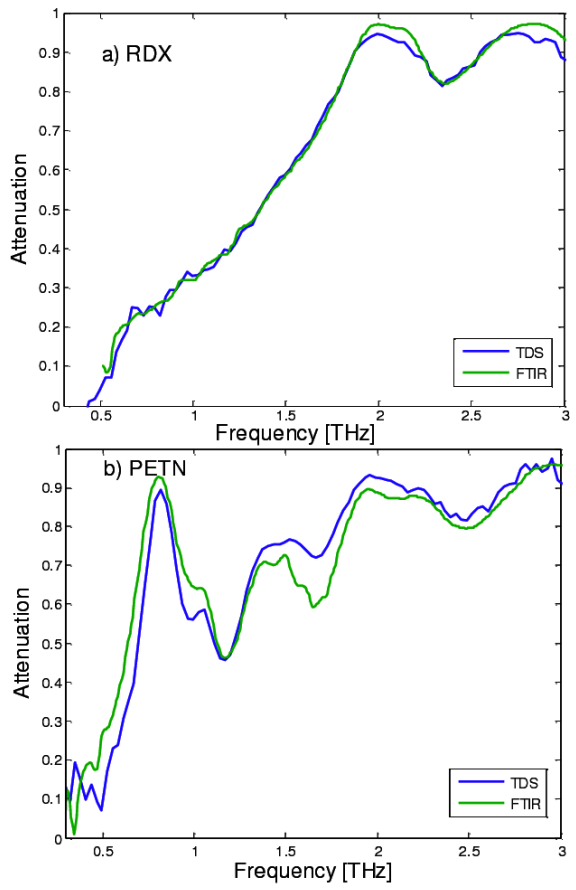

Fig. 4. Attenuation of RDX (a) and PETN (b) measured by TDS and FTIR.

The spectra measured by two methods are in good conformity taking into account shape of the spectra and po- 
sitions of characteristic peaks. Some discrepancies are connected with water vapor absorption and reflections in the TDS setup, which will be further optimized. The values of attenuation below $0.5 \mathrm{THz}$ are not accurate because this range is close to the limit of the system.

\section{Conclusions}

In this paper the sample preparation process, the TDS setup and results obtained for two commonly used explosives (hexogen and penthrite) are presented. The measured spectra were compared to FTIR results. Good conformity is observed. Obtained in this way characteristics can be used to create the data base of spectra of explosive materials.

\section{Acknowledgments}

The project is co-financed by the Polish Ministry of Science and Education under the project PRUE/31-436-2008-WAT.

\section{References}

[1] J. Chen, Y. Chen, H. Zhao, G. Bastiaans, X.-C. Zhang, Opt. Express 15, 12060 (2007).

[2] P. Struk, T. Pustelny, K. Gut, K. Golaszewska, E. Kaminska, M. Ekielski, I. Pasternak, E. Łusakowska, A. Piotrowska, Acta Phys. Pol. A 116, 414 (2009).

[3] C. Tyszkiewicz, A. Szpakowski, T. Pustelny, Acta Phys. Pol. A 116, 419 (2009).

[4] N. Palka, W. Ciurapinski, J. Wróbel, Acta Phys. Pol. A 116, 368 (2009).

[5] M.R. Leahy-Hoppa, M.J. Fitch, X. Zheng, L.M. Hayden, R. Osiander, Chem. Phys. Lett. 434, 227 (2007).

[6] W.M. Ciurapinski, M. Szustakowski, N. Palka, M. Zyczkowski, R. Ryniec, M. Piszczek, P. Zagrajek, Proc. SPIE 7485, 74850P (2009). 